\title{
Microbiota in Uterine Cavity of Unexplained Recurrent Spontaneous Abortion (URSA) Patients in Early Pregnancy
}

\section{Shiyu Bai}

Sun Yat-Sen University 2nd Affiliated Hospital: Sun Yat-Sen Memorial Hospital

\section{Shuai Fu}

Sun Yat-sen Memorial Hospital, Sun Yat-sen University https://orcid.org/0000-0003-1687-766X

\section{Liqiong Zhu}

Sun Yat-Sen University 2nd Affiliated Hospital: Sun Yat-Sen Memorial Hospital

\section{Manqi Chen}

Sun Yat-Sen University 2nd Affiliated Hospital: Sun Yat-Sen Memorial Hospital

\section{Bingqian Huang}

Sun Yat-Sen University 2nd Affiliated Hospital: Sun Yat-Sen Memorial Hospital

\section{Lihao Hu}

Sun Yat-Sen University 2nd Affiliated Hospital: Sun Yat-Sen Memorial Hospital https://orcid.org/00000001-7115-6326

\section{Hui Chen}

Sun Yat-Sen University 2nd Affiliated Hospital: Sun Yat-Sen Memorial Hospital Jianping Zhang ( $\nabla$ zjp2570@126.com )

Sun Yat-Sen University 2nd Affiliated Hospital: Sun Yat-Sen Memorial Hospital

\section{Research article}

Keywords: unexplained recurrent spontaneous abortion, microbiota, 16S rRNA sequencing

Posted Date: April 9th, 2021

DOI: https://doi.org/10.21203/rs.3.rs-402539/v1

License: (c) (1) This work is licensed under a Creative Commons Attribution 4.0 International License. Read Full License 
1 Brief title:

2 Microbiota in early pregnant uterine cavity of URSA

3 Title:

4 Microbiota in uterine cavity of unexplained recurrent spontaneous abortion (URSA)

5 patients in early pregnancy

\section{Authors and affiliations:}

7 Shiyu Bai ${ }^{1,2}$, Shuai $\mathrm{Fu}^{1}$, Liqiong Zhu ${ }^{1}$, Manqi $\mathrm{Chen}^{1}$, Bingqian Huang ${ }^{1,2}$, Lihao $\mathrm{Hu}^{1,2}$,

8 Hui Chen ${ }^{1}$, Jianping Zhang ${ }^{1}$

9 1.Department of Obstetrics and Gynecology, Sun Yat-sen Memorial Hospital, Sun Yat-sen

10 University, Guangzhou, 510120, Guangdong, China

11 2.Center for Reproductive Genetics and Reproductive Medicine, Sun Yat-sen Memorial Hospital,

12 Sun Yat-sen University, Guangzhou, 510120, Guangdong, China

13 Corresponding author:

14 Jianping Zhang, M.D., Ph.D., Professor

15 Address:

Department of obstetrics and gynecology, Sun Yat-sen Memorial Hospital, Sun

17 Yat-sen University, 107\# Yanjiang West Road, Guangzhou 510120, China

18 Tel: $+86-13902262443$

19 Email: zjp2570@126.com

20 Capsule:

21 Changes of Lactobacillus and Curvibacter dominated will lead to bad pregnancy

22 outcome. 


\section{Abstract}

Background: The majority of unexplained recurrent spontaneous abortion (URSA) was related to immune abnormalities. Inappropriate changes of microbiota could cause immune disorders. However, the role of uterine cavity microbiota in URSA has not be elucidated and few related studies are available for reference.

Methods: Using the double-lumen embryo transfer tubes to collect uterine cavity fluid samples from pregnant women in their first trimester. 16S rRNA sequencing was conducted to analysis the composition and abundance of the microbiota in samples.

Results: We enrolled 10 URSA cases and 28 induced miscarriage cases in their early pregnancy. Microbial communities were detected in all samples of URSA group $(100 \%, \mathrm{n}=10)$ vs. none of control group $(0 \%, \mathrm{n}=28)$. Two most dominant microbes are Lactobacillus and Curvibacter.

Conclusions:This study showed Lactobacillus and Curvibacter dominated colonizing in uterine cavity of URSA patients during early pregnancy and associated with URSA. Changes of dominant microbiota will lead to bad pregnancy outcome.

Keywords: unexplained recurrent spontaneous abortion, microbiota, 16S rRNA sequencing

\section{Background}

The human microbiota named the "second human genome" play a prominent role in human health and become the focus of scientific research with the assistance of gradual development of genome sequencing techniques(1). The human body and the human microbiota have become a symbiotic relationship and complex interaction occurs in almost every part of the body. The uterine cavity has always been considered a classic sterile cavity(2), contrary to this notion, a lot of studies showed that there were special microbial communities colonization in there(3-7). At the same time, the impact of the microbiota on reproductive system made us stand on the higher level to understand them.

Data have showed that the alterations of Lactobacilli dominance in uterus were linked to inflammation which result in spontaneous preterm birth or other adverse obstetric outcomes(1). A recent study suggested that the microbiota composition in endometrial fluid of patients receiving in vitro fertilization and embryo transfer (IVF-ET) may link to implantation and pregnancy rates(5, 8). However, the role of uterine cavity microbiota in URSA has not be elucidated and few related studies are available for reference. Therefore, the intention of our study was to determine the uterine cavity microbial composition in URSA patients and to understand the correlation between URSA and uterine cavity microbiota.

\section{Methods}

\section{Participants and sample collection}

A total of 38 pregnant women in their early pregnancy who came to Sun Yat-sen Memorial Hospital of Sun Yat-sen University were enrolled in our study. Then we divided them into two groups according to entry requirements (Figure 1). Those that meet the following conditions served as control group: needed an induced abortion for personal reasons this time and had no abortion history, no history of uterine cavity operation or vaginal infection on the day of surgery $(\mathrm{n}=28)$. B-ultrasound indicated that embryo was alive and there was no bleeding in uterine cavity or in vagina (Figure 1A). Others $(\mathrm{n}=10)$ were enrolled in URSA group: who were diagnosed as URSA, defined as the loss of $\geq 3$ consecutive pregnancies before 24 weeks' gestation(9) and the 
embryo had stopped development this time but had no bleeding in uterine cavity or in vagina through B-ultrasound. The chromosomal karyotype of the aborted embryos in the URSA group were normal. They all needed uterine curettage and had no vaginal infection on the day of surgery. After disinfection the vagina and cervix, excess liquid was cleansed by insertion cotton buds. Then a double-lumen embryo transfer tube (Cook Medical) was used to collect uterine cavity fluid carefully(9). When the outer sheath of the tube reached the junction between the endocervical canal and the uterine cavity, the inner tube was advanced into the uterine cavity for sample collection (Figure 1B). 8-10 $\mu \mathrm{l}$ uterine cavity fluid was transferred to a cryopreservation tube containing $1 \mathrm{ml}$ of RNase- and DNase-free water (Thermo Fisher Scientific) and snap-frozen in liquid nitrogen and then transferred to $-80^{\circ} \mathrm{C}$ for future analysis.
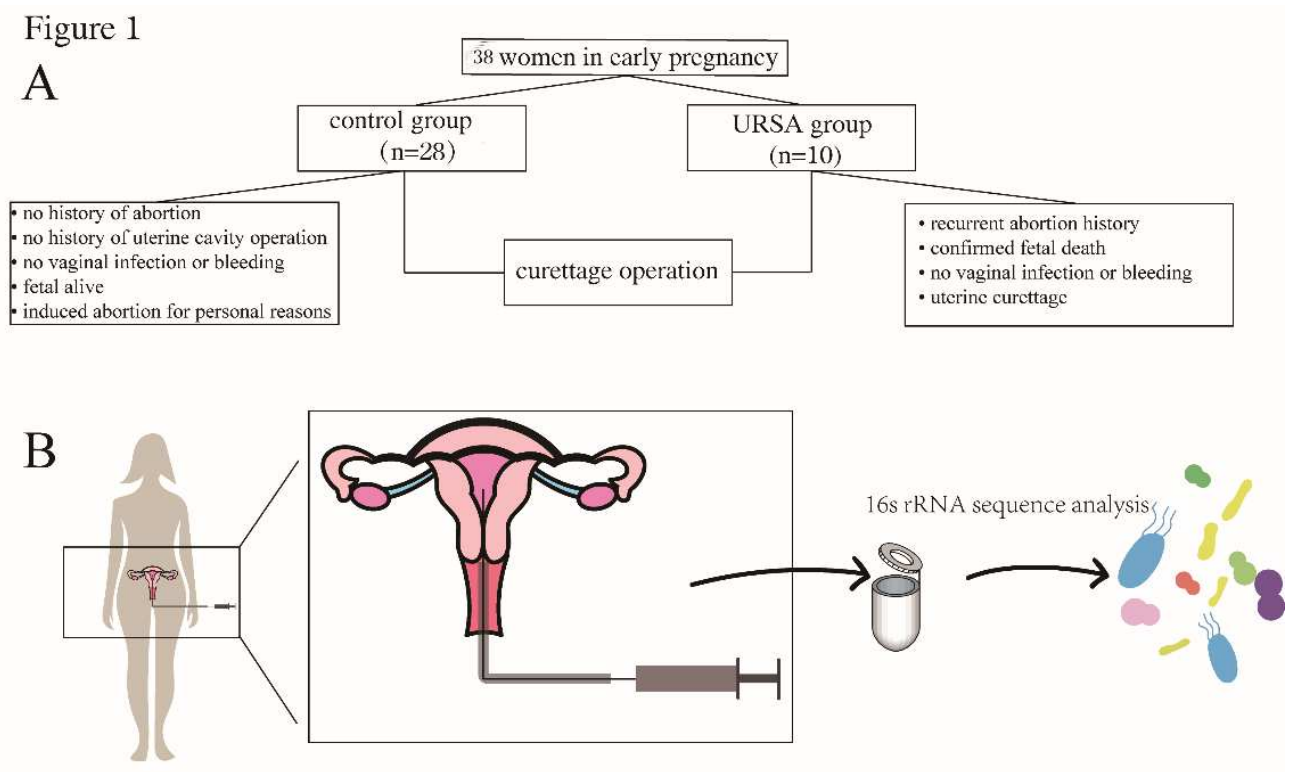

Figure 1. The enrolled criteria and sample collection method. (A) The workflow and enrolled criteria. (B) When the outer sheath of the tube reached the junction between the endocervical canal and the uterine cavity, the inner tube was advanced into the uterine cavity for collection 8-10 $\mu 1$ uterine cavity fluid. Then transferred the sample to a cryopreservation tube containing $1 \mathrm{ml}$ of RNase- and DNase-free water for future analysis.

\section{Genomic DNA extraction and 16S rRNA sequencing}

Isolation of genomic DNA from frozen uterine cavity fluid sample and the V3-V5 region of the 16S rRNA gene sequencing was completed at LC Sciences (Hangzhou, China) according to their standard service workflow. In brief, genomic DNA was extracted by QIAmp DNA mini-kit (Qiagen, USA). After 16S rRNA gene amplifcation by PCR following the Illumina protocols, the amplicons were sequenced using MiSeq ${ }^{\circledR}$ Reagent Kit v3 (Illumina) on a MiSeq-Illumina platform (Lifesequencing sequencing service, Valencia, Spain). PCR amplifcation and libraries controls were sequenced as negative controls to reduce sequencing error rate. We also used RNase- and DNase-free water as a blank control to remove background noise which may have impacted microbiota results.

\section{Bioinformatics and data analysis}

All the sequences were clustered into operational taxonomic units (OTU) based on their sequence similarity using QIIME, setting the sequence similarity threshold to 0.97 . Singletons and 
OTUs with a relative frequency below 0.01 were removed. Shannon was used to analysis Alpha diversity using QIIME. Beta diversity was done using Bray-Curtis distance and principal component analysis (PCA). Calypso software version 8.10.was used to perform the total statistical analysis on bacterial taxonomic identification.

Statistical analysis was performed using Graphpad Prism 8.0, and the measurement data were expressed as mean \pm standard deviation $(\mathrm{X} \pm \mathrm{SD})$. The mean comparison between groups was performed by $\mathrm{t}$-test. $\mathrm{P}<0.05$ was considered to be statistically significant.

\section{Results}

The general characteristics of the participants were listed in Table 1 including age, gestational week, history of uterine cavity operation and reproductive tract infection. The mean age of control group was 28.96 years old, which was much younger than URSA group $(P=0.0272)$. Significant differences were not found in gestational week $(5.89 \pm 0.19, \mathrm{n}=28$ vs $6.46 \pm 0.39, \mathrm{n}=10$, $P=0.1422$ ). None of the control group had the uterine operation history while all participants of the URSA group had history of uterine cavity operations such as uterine curettage, hysteroscopy, etc. None participant had bacterial vaginosis before surgery. $70 \%$ of URSA group ever had a live birth.

Table 1 General characteristics of participants

\begin{tabular}{cccc}
\hline & control group $(\mathrm{n}=28)$ & URSA group $(\mathrm{n}=10)$ & $P$ value \\
\hline age(years), mean \pm SD & $28.96 \pm 1.10$ & $33.08 \pm 1.13$ & 0.0272 \\
Gestational week(weeks), mean \pm SD & $5.89 \pm 0.19$ & $6.46 \pm 0.39$ & 0.1422 \\
History of uterine cavity & $0(0 \%)$ & $10(100 \%)$ & - \\
operation,no.(\%) & $1(3.57 \%)$ & $0(0 \%)$ & - \\
Reproductive tract infection,no.(\%) & $14(50 \%)$ & $10(100 \%)$ & 0.005 \\
Ever pregnant,no.(\%) & $9(32.14 \%)$ & $7(70 \%)$ & 0.037 \\
Ever had a live birth,no.(\%) & $0(0 \%)$ & $10(100 \%)$ & - \\
History of miscarriage,no.(\%) & &
\end{tabular}

Surprisingly, all uterine cavity fluid samples of URSA group $(\mathrm{n}=10)$ showed positive detection of microbial communities by 16s rRNA sequencing analysis, while none sample showed positive detection from control group (Figure 2). After qualify negative sample, a total of 488,380 reads were obtained. The average of operational taxonomic units (OTUs) per uterine cavity fluid sample observed was 90.5. The valid reads were assigned to 84 OTUs in URSA group. The Alpha diversity measured by Shannon diversity, Chao 1 index and Simpson index were shown in Table 2.

Next, we analyzed the microbial communities. As seen in Figure 3, we found that Firmicuteswas the most dominant phylum in samples of URSA 4, 7, 8 and $9(74.02,97.49,55.28$ and $81.09 \%$; respectively); followed by Proteobacteria which was the most dominant phylum in samples of URSA 5, $10(64.09,81.22 \%)$. Deinococcus-Thermus was the most abundant in samples of URSA 3, $6(96.92,93.55 \%)$. Actinobacteria $(67.92 \%)$ was dominant in samples of URSA 1. We further analyzed the relative abundance of genus in each sample. As shown in Figure 4, Lactobacillus were higher in URSA 4, 7, 8, 9 (72.50, 96.83, 52.68, 79.29\%; respectively) and Ureaplasma were higher in URSA 3, 6 (96.92, 93.55\%). Obviously, Curvibacter was the subdominantbacteria. 
Table 2 Microbiota community $\alpha$ diversity with URSA samples

\begin{tabular}{ccccccc}
\hline Samples & $\begin{array}{c}\text { Number of } \\
\text { raw reads }\end{array}$ & $\begin{array}{c}\text { Number of } \\
\text { valid reads }\end{array}$ & $\begin{array}{c}\text { Observed } \\
\text { OTUs }\end{array}$ & $\begin{array}{c}\text { Chao 1 } \\
\text { index }\end{array}$ & $\begin{array}{c}\text { Shannon } \\
\text { index }\end{array}$ & $\begin{array}{c}\text { Simpson } \\
\text { index }\end{array}$ \\
\hline URSA1 & 87517 & 76526 & 78 & 78.20 & 3.53 & 0.80 \\
\hline URSA2 & 80588 & 77217 & 60 & 61.75 & 1.00 & 0.28 \\
\hline URSA3 & 83582 & 80623 & 105 & 65.80 & 1.28 & 0.52 \\
\hline URSA4 & 52897 & 46516 & 111 & 111.00 & 3.13 & 0.70 \\
\hline URSA5 & 82413 & 73825 & 113 & 116.00 & 5.70 & 0.95 \\
\hline URSA6 & 85788 & 82415 & 51 & 53.14 & 1.46 & 0.56 \\
\hline URSA7 & 83205 & 78248 & 105 & 110.08 & 1.11 & 0.28 \\
\hline URSA8 & 83032 & 70919 & 91 & 91.00 & 3.59 & 0.74 \\
\hline URSA9 & 86105 & 72305 & 96 & 96.00 & 1.87 & 0.38 \\
\hline
\end{tabular}

Table 3 the abundance Top 3 bacteria in genus level and the gestational week of surgery or embryo damage of all samples

\begin{tabular}{cclll}
\hline Sample & $\begin{array}{c}\text { Gestational week of } \\
\text { surgery or embryo } \\
\text { damage(weeks) }\end{array}$ & Top 1 bacteria & Top 2 bacteria & Top 3 bacteria \\
\hline URSA1 & 5 & Gardnerella & Curvibacter & Acinetobacter \\
URSA2 & 6 & Sneathia & Anaerococcus & Ureaplasma \\
URSA3 & 6 & Ureaplasma & Lactobacillus & Curvibacter \\
URSA4 & $6+$ & Lactobacillus & Curvibacter & Gardnerella \\
URSA5 & $6+$ & Curvibacter & Ralstonia & Prevotella \\
URSA6 & 7 & Ureaplasma & Lactobacillus & Gardnerella \\
URSA7 & $7+$ & Lactobacillus & Curvibacter & Bradyrhizobium \\
\hline URSA8 & $8+$ & Lactobacillus & Curvibacter & Acinetobacter \\
\hline URSA9 & $8+$ & Lactobacillus & Curvibacter & Sphingobium \\
URSA10 & $9+5$ & Curvibacter & Bradyrhizobium & Rubellimicrobium \\
\hline
\end{tabular}
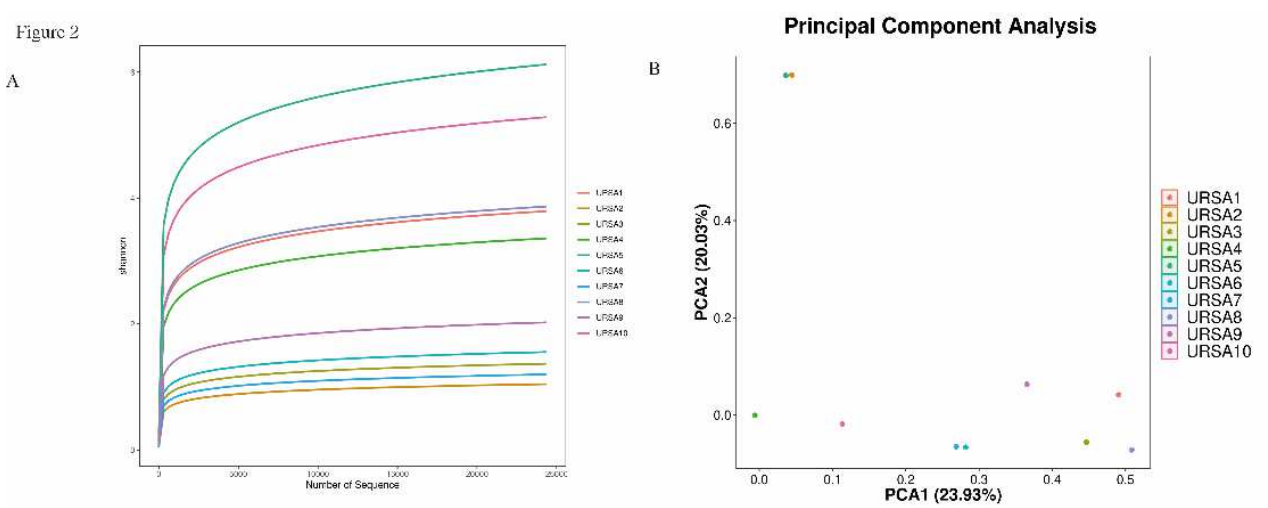
150 Figure 2. 16S rRNA sequencing analysis of microbial communities. (A) Alpha diversity was

151 measured by Shannon index. (B)Beta diversity was showed by principal component analysis 152 (PCA).

Figure 3



Figure 4

154 Figure 3. The composition of microbiome in Phylum level of all samples.



Figure 4. The composition of microbiome in Genus level of all samples. 
Then, we listed the Top 3 abundant bacteria in genus level and the gestational week of embryo damage of all samples (Table 3). Lactobacillus or Curvibacter was the most dominant in most samples. The gestational week of embryo damage of samples which dominated by Lactobacillus was later than that dominated by other bacteria from URSA group $(7.25 \pm 0.48$ vs $6.50 \pm 0.56, \mathrm{P}=0.375)$.

\section{Discussion}

To the best of our knowledge, this is the first study to detect the microbiota in uterine cavity in first-term pregnancy. Due to the limited availability of materials, most studies focused on the microbial communities in uterine cavity in the unpregnant state $(3,7,8,10,11)$. And some other studies put their attention on the microbiome of amniotic fluid and placenta(10, 12, 13). Therefore, the status of the uterine cavity microbiota in pregnancy has yet to be elucidated.

Embryo chromosomal abnormalities were one of the most relevant causes of miscarriage, so our karyotype analysis of aborted embryos in the URSA group eliminated this important confounding factor. To further ensure the credibility of our findings, we attempted to rule out the possibility of sample contamination from cervix and vagina meticulously. After disinfection before surgery, we used a sterile and sleeved embryo transfer tube for sample collection. In order to avoid contamination of blood, the patient would be rejected once she had vaginal bleeding or bleeding in the uterine cavity through B-ultrasound. Therefore, the possibility of false positives in our results was very minimal.

In our study, we recognized that, in first-term pregnant uterine cavity of URSA women whose embryo stopped developing, there were microbiome such as Firmicutes, Proteobacteria, Deinococcus-Thermus, Lactobacillus, etc. It is in line with several previous studies which focused on microbiome colonization in female upper genital tract $(6,7)$. Altered microbiota in uterine cavity may be associate with URSA. A study showed that there were distinct differences in the relative abundance of microbial groups between URSA and control group on family and genus levels. URSA samples showed increased abundance of Firmicutes, which was consistent with our research results(9). Lactobacillus was a dominated resident microbiota in healthy endometrium and several studies detected it may be associated with infertility, endometriosis, chronic endometritis, endometrial polyps and dysfunctional menstrual bleeding(14, 15). It also was the most dominated microbiota in the vagina. And Brown et al. found that reduced relative abundance of Lactobacillus is associated with premature cervical dilation(16). We compared the cases and found that the gestational week of embryo damage of URSA patients whose uterine cavity dominated by the bacteria was later than others. This may indicate Firmicutes-Lactobacillus domination may be conducive to embryo development. We did not find Lactobacillus colonized in control group and we thought that they may only exist in unpregnant uterine cavity and disappear during early pregnancy in normal females.

Mounting studies showed that Ureaplasma was a common microbiota which contribute to bad pregnancy outcomes like preterm delivery and chronic inflammation of the productive tract(17-19). A recent review focused on the correlation between Ureaplasma exposure and the important morbidities of prematurity and got conclusion that Ureaplasma was attributed to neonatal morbidities of bronchopulmonary dysplasia, intraventricular hemorrhage, and necrotizing enterocolitis(20). Another pilot study showed that infertile women were more likely had Ureaplasma in the vagina(21). In our study, Ureaplasma was dominant in URSA 3 and 6, and the Top 3 highest abundance in URSA 2. Therefore, we speculate that increased abundance of 
Ureaplasma may lead to worse early pregnancy outcomes. We guessed that different dominant microbiome will lead to different pregnancy outcome. In some samples of URSA group, we also found some other reproductive tract pathogens like Gardnerella, Anaerococcus, etc. The most likely reason was that these patients had multiple uterine cavity operations before, and these pathogens ascended through the lower reproductive tract and then colonized in the uterine cavity, which had a negative impact on embryo implantation and development.

Curvibacter being dominant or predominant microbe was detected in almost all samples of URSA group in our study. It was previously isolated from various aqueous environments and had identified in atherosclerotic plaques(22). Curvibacter was first reported existed in the amniotic fluid and vaginal fluid microbiota of healthy pregnant women in a recent study (23). But there was no Curvibacter existed in the control group in our study, so whether it be harmful to pregnancy still need to discuss in the future.

It has been suggested that fetus development needs a fertile environment(24). Interestingly, in our study, all samples in control group were sterile of microbiota. A study showed that $40 \%$ of the endometrial samples obtained by abdominal hysterectomy detected no microbiome(25). This exciting phenomenon raises doubts whether uterine microbiome colonization in all early pregnant women or just in URSA women, whether the detected microbiota is in the uterus temporarily or permanently, or whether they are just contamination(26). Therefore, in the near future, we will concentrate on a series of related work to verify our assumption that there may be a "microbiota-containing time window" in the uterine cavity in the early pregnancy. As the pregnancy progresses, the uterine cavity gradually changes from a microbiota-containing state to a sterile state. In the latter part of pregnancy, it becomes a bacterial fertile state again. Perhaps, lengthened or shortened "microbiota-containing time window" may lead to severe pregnancy outcomes.

Our research still has some limitations should be considered. Firstly, in the materials of 28 uterine cavity fluid samples of control group, none sample was microbiome positive that lead to statistical analysis of differences of diversity and abundance between two groups unavailable. A small sample of negative data may not represent the whole truth. We cannot rule out the influence of previous uterine cavity operation history on the uterine cavity flora as a confounding factor. Secondly, our data just showed the functions of whole microbiota instead of the dominant ones so that how they affect URSA is not clear. And the causal relationship between the uterine cavity flora and the abortion is difficult to determine.

\section{Conclusion}

In summary, our study showed an interesting finding of a phenomenon that microbiota could be found in the pregnant uterine cavity of URSA patients. We speculate that either the presence of microbiota leads to URSA or this could corroborate the theory of uterine fertile of microbiota. Nevertheless, studies are still needed to replicate these initial findings to either confirm the interesting phenomenon or to open a new field of the existence and function of microbiota in pregnant uterine which may be neglected. Questions of which microbiota is the real culprit of URSA and whether there is a "microbiota-containing time window" for microbiota in the uterine cavity remains to be elucidated in future studies.

\section{Abbreviations}

unexplained recurrent spontaneous abortion (URSA)

in vitro fertilization and embryo transfer (IVF-ET) 
operational taxonomic units (OTU)

principal component analysis (PCA)

\section{Declarations}

\section{Ethics approval and consent to participate}

Approval for our study was obtained from the Ethics Committee of Sun Yat-sen Memorial Hospital of Sun Yat-sen University (SYSEC-KY-KS-2020-146) and written informed consent was obtained from all participants.

\section{Consent for publication}

All of the authors have agreed to publish this manuscript and signed the consent.

\section{Availability of data and material}

The datasets used and analysed during the current study available from the corresponding author on reasonable request.

\section{Competing interests}

No conflicts of interest in this study.

\section{Funding}

This study was supported by grants from the National Natural Science Foundation of China (grant nos. 81771660 and 81741017), the Science and Technology Planning Project of Guangdong Province (2017A020214018 and 2017A020214003), the Guangdong Natural Science Foundation (2018A030313023 and 2018A030310162), the Science and Technology Planning Project of Guangzhou City Central Universities (201704020034); the 5010 projects of Sun Yat-Sen University (2012006), and the Fundamental Research Funds for the Central Universities (Youth Program12ykpy29).

\section{Authors' contributions}

Shiyu Bai performed research development and wrote the manuscript; Shuai Fu performed data collection and manuscript editing; Manqi Chen, Bingqian Huang and Lihao Hu performed project development and data collection; Hui Chen and Jianping Zhang performed the supervision, data interpretation and manuscript revision. All the authors reviewed the manuscripts.

\section{Acknowledgment}

The authors thank all participants in this study. The authors also thank Yilei Zhong, Yanli Shen for assistance with material purchase and sample collection. We also be grateful to Meilan Liu, Lili Meng for helpful discussion.

\section{Figure legends}

Table 1 General characteristics of participants

Table 2 Microbiota community $\alpha$ diversity with URSA samples

Table 3 the abundance Top 3 bacteria in genus level and the gestational week of surgery or embryo damage of all samples

Figure 1. The enrolled criteria and sample collection method. (A) The workflow and enrolled criteria. (B) When the outer sheath of the tube reached the junction between the endocervical canal and the uterine cavity, the inner tube was advanced into the uterine cavity for collection 8-10 $\mu 1$ uterine cavity fluid. Then transferred the sample to a cryopreservation tube containing $1 \mathrm{ml}$ of RNase- and DNase-free water for future analysis.

Figure 2. 16S rRNA sequencing analysis of microbial communities. (A) Alpha diversity was measured by Shannon index. (B)Beta diversity was showed by principal component analysis (PCA). 
Figure 3. The composition of microbiome in Phylum level of all samples. Figure 4. The composition of microbiome in Genus level of all samples.

\section{References}

1. Franasiak JM, Scott RT, Jr. Introduction: Microbiome in human reproduction. Fertil Steril. 2015;104(6):1341-3.

2. Evans J, Salamonsen LA, Winship A, Menkhorst E, Nie G, Gargett CE, et al. Fertile ground: human endometrial programming and lessons in health and disease. Nat Rev Endocrinol. 2016;12(11):654-67.

3. Baker JM, Chase DM, Herbst-Kralovetz MM. Uterine Microbiota: Residents, Tourists, or Invaders? Front Immunol. 2018;9:208.

4. Chen HJ, Gur TL. Intrauterine Microbiota: Missing, or the Missing Link? Trends Neurosci. 2019;42(6):402-13.

5. Moreno I, Codoner FM, Vilella F, Valbuena D, Martinez-Blanch JF, Jimenez-Almazan J, et al. Evidence that the endometrial microbiota has an effect on implantation success or failure. Am J Obstet Gynecol. 2016;215(6):684-703.

6. Peric A, Weiss J, Vulliemoz N, Baud D, Stojanov M. Bacterial Colonization of the Female Upper Genital Tract. Int J Mol Sci. 2019;20(14).

7. Tomaiuolo R, Veneruso I, Cariati F, D'Argenio V. Microbiota and Human Reproduction: The Case of Female Infertility. High Throughput. 2020;9(2).

8. Kitaya K, Nagai Y, Arai W, Sakuraba Y, Ishikawa T. Characterization of Microbiota in Endometrial Fluid and Vaginal Secretions in Infertile Women with Repeated Implantation Failure. Mediators Inflamm. 2019;2019:4893437.

9. Liu Y, Wong KK, Ko EY, Chen X, Huang J, Tsui SK, et al. Systematic Comparison of Bacterial Colonization of Endometrial Tissue and Fluid Samples in Recurrent Miscarriage Patients: Implications for Future Endometrial Microbiome Studies. Clin Chem. 2018;64(12):1743-52.

10. Seo SS, Arokiyaraj S, Kim MK, Oh HY, Kwon M, Kong JS, et al. High Prevalence of Leptotrichia amnionii, Atopobium vaginae, Sneathia sanguinegens, and Factor 1 Microbes and Association of Spontaneous Abortion among Korean Women. Biomed Res Int. 2017;2017:5435089.

11. Vilella F, Ramirez L, Berlanga O, Martinez S, Alama P, Meseguer M, et al. PGE2 and PGF2alpha concentrations in human endometrial fluid as biomarkers for embryonic implantation. J Clin Endocrinol Metab. 2013;98(10):4123-32.

12. Leiby JS, McCormick K, Sherrill-Mix S, Clarke EL, Kessler LR, Taylor LJ, et al. Lack of detection of a human placenta microbiome in samples from preterm and term deliveries. Microbiome. 2018;6(1):196.

13. Tuominen H, Rautava S, Syrjanen S, Collado MC, Rautava J. HPV infection and bacterial microbiota in the placenta, uterine cervix and oral mucosa. Sci Rep. 2018;8(1):9787.

14. Pelzer ES, Willner D, Buttini M, Huygens F. A role for the endometrial microbiome in dysfunctional menstrual bleeding. Antonie van Leeuwenhoek. 2018;111(6):933-43.

15. Hernandes C, Silveira P, Rodrigues Sereia AF, Christoff AP, Mendes H, Valter de Oliveira LF, et al. Microbiome Profile of Deep Endometriosis Patients: Comparison of Vaginal Fluid, Endometrium and Lesion. Diagnostics (Basel). 2020;10(3).

16. Brown RG, Chan D, Terzidou V, Lee YS, Smith A, Marchesi JR, et al. Prospective observational study of vaginal microbiota pre- and post-rescue cervical cerclage. BJOG. 2019;126(7):916-25.

17. Sweeney EL, Dando SJ, Kallapur SG, Knox CL. The Human Ureaplasma Species as Causative Agents of Chorioamnionitis. Clin Microbiol Rev. 2017;30(1):349-79. 
18. Sweeney EL, Kallapur SG, Gisslen T, Lambers DS, Chougnet CA, Stephenson SA, et al. Placental Infection With Ureaplasma species Is Associated With Histologic Chorioamnionitis and Adverse Outcomes in Moderately Preterm and Late-Preterm Infants. J Infect Dis. 2016;213(8):1340-7.

19. Cregger MA, Lenz K, Leary E, Leach R, Fazleabas A, White B, et al. Reproductive Microbiomes: Using the Microbiome as a Novel Diagnostic Tool for Endometriosis. Reproductive Immunology. 2017. 20. Viscardi RM. Ureaplasma species: role in neonatal morbidities and outcomes. Arch Dis Child Fetal Neonatal Ed. 2014;99(1):F87-92.

21. Wee BA, Thomas M, Sweeney EL, Frentiu FD, Samios M, Ravel J, et al. A retrospective pilot study to determine whether the reproductive tract microbiota differs between women with a history of infertility and fertile women. Aust N Z J Obstet Gynaecol. 2018;58(3):341-8.

22. Ziganshina EE, Sharifullina DM, Lozhkin AP, Khayrullin RN, Ignatyev IM, Ziganshin AM. Bacterial Communities Associated with Atherosclerotic Plaques from Russian Individuals with Atherosclerosis. PLoS One. 2016;11(10):e0164836.

23. He Q, Kwok LY, Xi X, Zhong Z, Ma T, Xu H, et al. The meconium microbiota shares more features with the amniotic fluid microbiota than the maternal fecal and vaginal microbiota. Gut Microbes. 2020;12(1):1794266.

24. Benner M, Ferwerda G, Joosten I, van der Molen RG. How uterine microbiota might be responsible for a receptive, fertile endometrium. Human Reproduction Update. 2018;24(4):393-415. 25. Winters AD, Romero R, Gervasi MT, Gomez-Lopez N, Tran MR, Garcia-Flores V, et al. Does the endometrial cavity have a molecular microbial signature? Sci Rep. 2019;9(1):9905.

26. Molina NM, Sola-Leyva A, Saez-Lara MJ, Plaza-Diaz J, Tubic-Pavlovic A, Romero B, et al. New Opportunities for Endometrial Health by Modifying Uterine Microbial Composition: Present or Future? Biomolecules. 2020;10(4). 


\section{Figures}
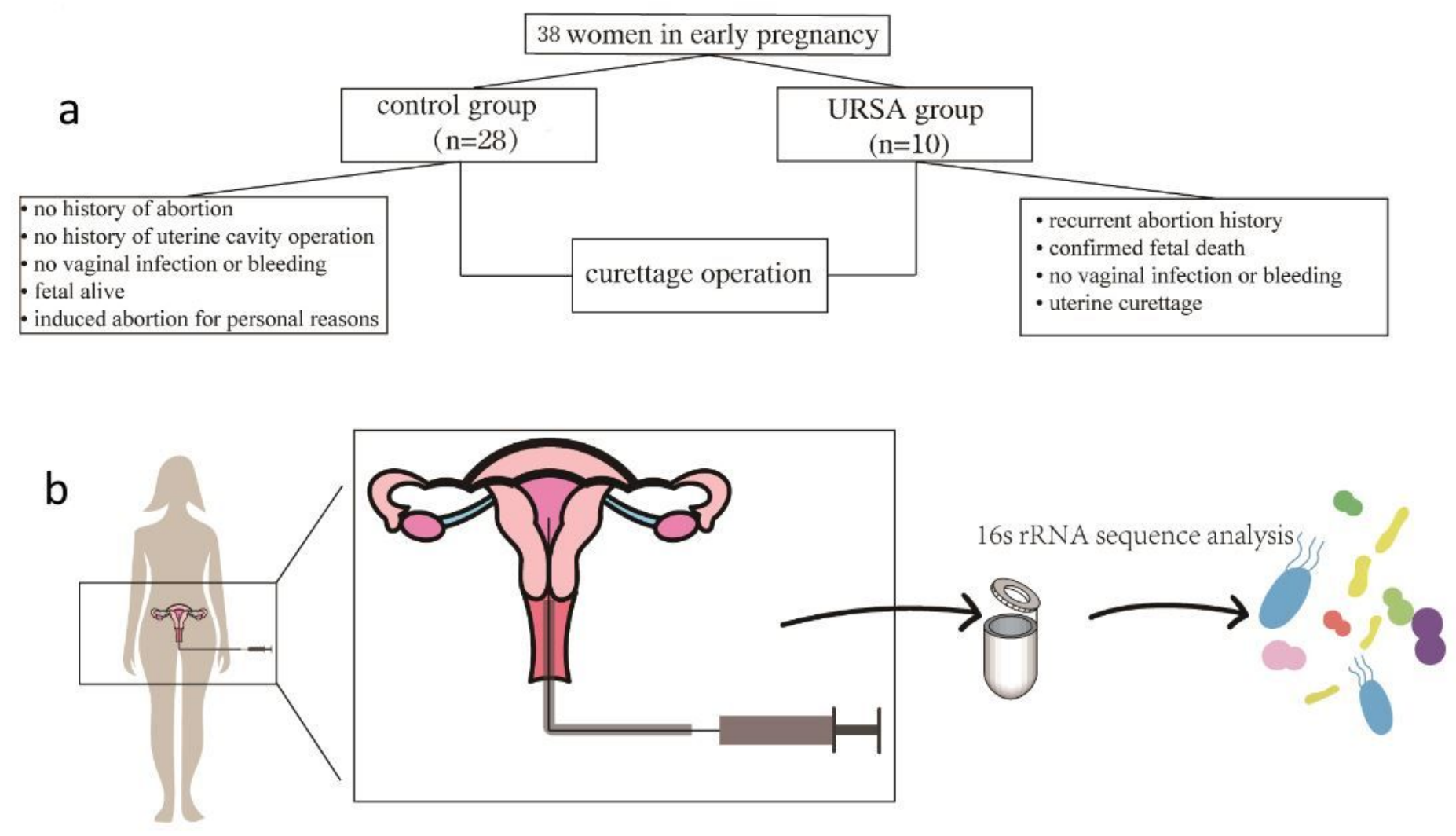

\section{Figure 1}

The enrolled criteria and sample collection method. (A) The workflow and enrolled criteria. (B) When the outer sheath of the tube reached the junction between the endocervical canal and the uterine cavity, the inner tube was advanced into the uterine cavity for collection 8-10 $\mu$ uterine cavity fluid. Then transferred the sample to a cryopreservation tube containing $1 \mathrm{ml}$ of RNase- and DNase-free water for future analysis. 

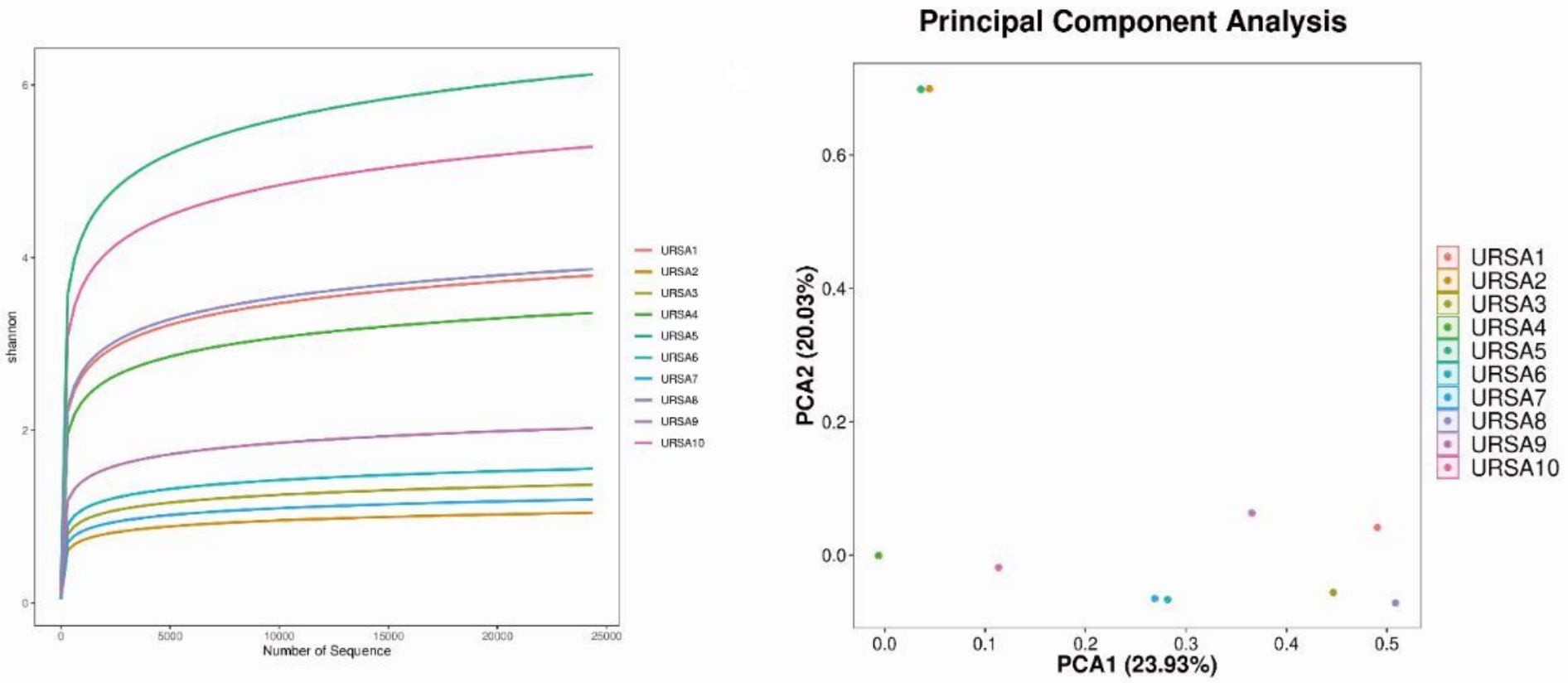

Figure 2

16S rRNA sequencing analysis of microbial communities. (A) Alpha diversity was measured by Shannon index. (B)Beta diversity was showed by principal component analysis (PCA). 
Phylum

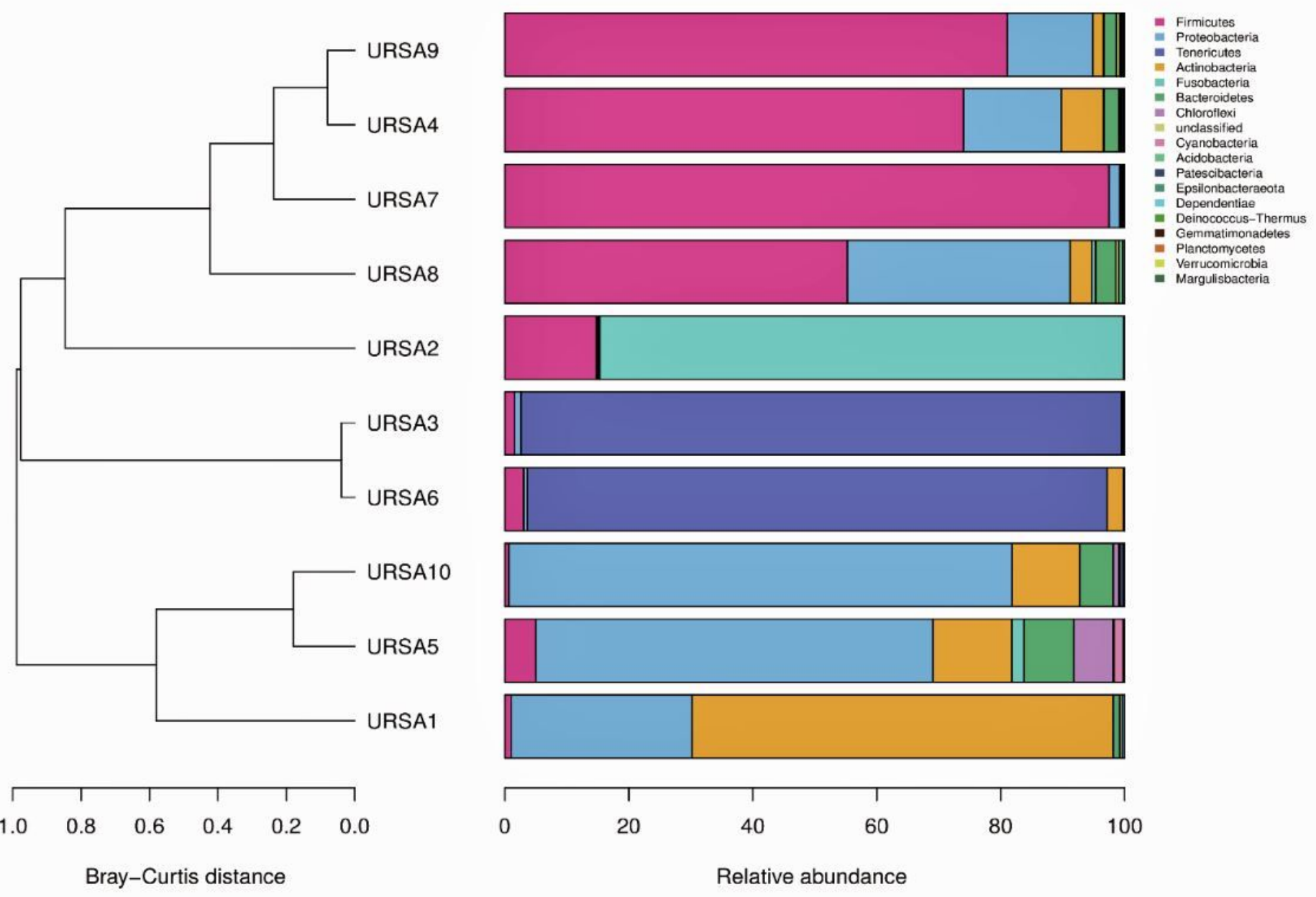

\section{Figure 3}

The composition of microbiome in Phylum level of all samples. 


\section{Genus}


Lactobscillus Ureaplasma Sncathia

Gurvibacter

Bracyrhizobium

- Acinetobacter

- Rubellimicrobium

Sphingorrorias

Anearcocaccus

Sediminibacterum

Enrydrobacter

Sohingobium

Methylobacteriurn

Prevotalla

Ralstonia

- Blastococcus

Burkholdoriaks

Pseudomonas

- Deltia

Pelamonas

Perchroracter

Shuttleworthis

Aquabarium

- Mobiluncus

Phodococcus

Metrylophilus

Chloroflaxi undassfied

Others


Bray-Curtis distance

Relative abundance

Figure 4

The composition of microbiome in Genus level of all samples. 